\title{
Desempenho operacional e econômico do terraceamento de pequenas propriedades com arados de discos ${ }^{1}$
}

\author{
Tiago Pereira da Silva Correia ${ }^{2}$; Vinicius Paludo ${ }^{3}$; Paulo Roberto Arbex Silva ${ }^{4}$; Saulo \\ Fernando Gomes de Sousa ${ }^{5}$; Patrícia Pereira Dias ${ }^{6}$; Bruna Dionofre ${ }^{7}$; Karen Pereira da Silva \\ Carneiro $^{8}$
}

\begin{abstract}
Resumo: O terraceamento com arados de discos ainda é prática comum em pequenas propriedades rurais, munidas de implementos e tratores de menor porte. O objetivo do trabalho foi avaliar o desempenho operacional e econômico de diferentes modelos de arados de discos comumente usados na construção de terraços em pequenas propriedades rurais. $\mathrm{O}$ experimento foi realizado com arados de discos e tratores em três pequenas propriedades rurais nos municípios de Botucatu-SP e Avaré-SP. O delineamento experimental utilizado foi o inteiramente casualizado (DIC) com quatro repetições, sendo os tratamentos: arado fixo de três discos (AF3D), arado fixo de dois discos (AF2D), arado reversível de três discos (AR3D), e arado reversível de dois discos (AR2D). Os terraços foram construídos com base estreita em áreas com declive entre 13 e $16 \%$ e utilizadas como pastagem. As variáveis avaliadas foram: eficiência operacional (Ef), capacidade de campo efetiva (Cce), consumo horário (Ch) e operacional de combustível (Cct), custo horário $(\mathrm{CH})$ e operacional $(\mathrm{COT})$ e eficiência econômica (EFec). Os resultados indicaram que arados reversíveis apresentam maior Ef e Cce, arado AF3D maior $\mathrm{Ch}$ e Cct, e economicamente o AR2D possibilita menores custos e maior EFec.
\end{abstract}

Palavras-chave: Conservação do solo; Eficiência operacional; Capacidade de campo; Consumo de combustível; Custos.

\section{Operational and economic performance of terracing small properties with disc plow}

\begin{abstract}
The terracining is a common practice in some small farms, equipped with implements and small tractors. The purpose of the work was to evaluate the operational and economic performance of different models of disc plows commonly used for building terraces on small farms. The experiment using three tipes of disc plows and tractors small are done in the cities of Botucatu-SP and Avaré-SP. The study was conduct in a completely randomized experiment (DIC) with four replications, being the treatments: two fixed plow (AF2D), two discs reversible (AR2D), three fixed discs plow (AF3D) and three reversible discs plow (AR3D).Terraces were built on contour planting in areas with between 13 and $16 \%$ used for pasturing. The variables evaluated were: efficiency operational efficiency (Eff), effective field capacity (Cce), hourly consumption (Chc) and operational (Cct), hourly $(\mathrm{CH})$ and operational cost (TOC), and economic efficiency (EFec). The results indicated that the reversible plows present higher Ef and Cce, The AF3D showed the higher Chc and Cct, but economically, the AR2D resulted in the lower costs and higher EFec.
\end{abstract}

Keywords: Conservation of soil, Operational efficiency, Field capacity, Fuel consumption, Costs.

\footnotetext{
${ }^{1}$ Submetido em 10/11/2017 e aprovado em 12/01/2018

${ }^{2}$ Doutor em Agronomia; Professor adjunto, Universidade de Brasília (UnB), Faculdade de Agronomia e Medicina Veterinária, Brasília-DF, CEP: 70910-900; E-mail: tiagocorreia@unb.br

${ }^{3}$ Mestre em Agronomia; Doutorando, Universidade Estadual Paulista (UNESP), Programa de Pós-Graduação em Agronomia, Botucatu-SP, CEP: 18610-307; E-mail: vipaludo@gmail.com

${ }^{4}$ Doutor em Agronomia; Professor Assistente, Universidade Estadual Paulista (UNESP), Faculdade de Ciências Agronômicas, Botucatu-SP, CEP: 18610-307; E-mail: arbex@fca.unesp.br

${ }^{5}$ Doutor em Agronomia, Agroefetiva Serviços Ss Ltda, Botucatu-SP, CEP: CEP 18.603-760; E-mail: saulofgs@ @otmail.com

${ }^{6}$ Doutora em Agronomia; Pós-Doutoranda, Universidade Estadual Paulista (UNESP), Botucatu-SP, CEP: 18610-307; E-mail: eng.amb.patricia@gmail.com

${ }^{7}$ Graduando em Agronomia, Universidade de Brasília (UnB), Faculdade de Agronomia e Medicina Veterinária, Brasília-DF, CEP: 70910-900; E-mail: bruna_dionofre@yahoo.com

${ }^{8}$ Graduando em Agronomia, Universidade de Brasília (UnB), Faculdade de Agronomia e Medicina Veterinária, Brasília-DF, CEP: 70910-900; E-mail: karenpereira0@gmail.com
} 


\section{Introdução}

A erosão dos solos é um sério e crescente problema nas propriedades rurais brasileiras, ocasionando perdas anuais de 600 milhões de toneladas de solo agrícola (Cunha et al., 2011). Segundo Panachuki et al. (2006), na maioria das vezes a erosão ocorre por desproteção do solo às chuvas e ventos intensos, sendo os principais fatores de depauperamento acelerado da fertilidade do solo, acarretando aumento dos custos de produção e redução de produtividade. De acordo com Dechen et al. (2015), as necessidades dos produtores em repor fertilidade aos solos erodidos são expressos por valores da ordem de até US\$107,76 por ha $\mathrm{ano}^{-1}$, representando custo agrícola brasileiro na ordem de US $\$ 1,3$ bilhão ao ano.

Várias são as práticas utilizadas para controlar a erosão dos solos por água da chuva, sendo as mais comuns os preparos conservacionistas do solo, cultivo mínimo, plantio direto e o terraceamento mecânico (Ferreira et al., 2010). Terraços são estruturas compostas por um dique e um canal, dispostos no sentido transversal à declividade do terreno, formando obstáculos físicos para reduzir a velocidade do escoamento superficial e ordenar o movimento da água sobre a superfície do solo (Magalhães, 2013).

Apesar de ser uma prática antiga o terraceamento apresenta dificuldades relativas à sua construção e manutenção, em especial nas pequenas propriedades rurais. De acordo com Vasconcelos et al. (2013), nestas é comumente constatado menor recurso tecnológico do maquinário agrícola, tratores e implementos de potência, dimensões e quantidades, e limitações de uso por desconhecimento técnico de regulagens, adequação ao trabalho, dimensionamento e estudo econômico.

Segundo descreve Melo et al. (2016) para que o terraceamento funcione bem, principalmente para agricultores com menor infraestrutura mecanizada e até aporte financeiro, é importante conhecer a capacidade de trabalho e limitações das máquinas disponíveis. A combinação desses fatores pode elevar custos financeiros e não obter a eficiência quantitativa e qualitativa da operação (Silva e Voltarelli, 2015).

De acordo com Melo et al. (2016) em propriedades com maior infraestrutura de máquinas e área cultivada, os terraços são construídos predominantemente com arado terraceador tracionado por tratores de maior potência, obtendo-se elevada largura de trabalho, velocidade e capacidade produtiva. Entretanto, em pequenas propriedades é comum a utilização de arados de discos e tratores de baixa potência, entre 50 e $100 \mathrm{cv}$.

O objetivo do trabalho foi avaliar o desempenho operacional e econômico de diferentes modelos de arados de discos comumente usados na construção de terraços em pequenas propriedades rurais.

\section{Material e Métodos}

$\mathrm{O}$ experimento foi realizado em três pequenas propriedades rurais do estado de São Paulo, uma localizada no município de Botucatu e duas no município de Avaré. As propriedades de Avaré, identificadas como P1 e P2, baseiam-se na produção familiar de hortícolas, citros e pecuária leiteira, constituídas respectivamente por áreas de 12,7 e 14 ha. A propriedade de Botucatu, identificada como P3, trata-se de um sítio modelo da Faculdade de Ciências Agrárias da Universidade Estadual Paulista (FCA/UNESP), unidade demonstrativa de aproximadamente 15 ha destinados a experimentação agrícola e extensão rural.

$\mathrm{O}$ delineamento experimental utilizado foi o inteiramente casualizado (DIC), constituído por quatro tratamentos: arado fixo de três discos (AF3D), arado fixo de dois discos (AF2D), arado reversível de três discos (AR3D) e arado reversível de dois discos (AR2D), com quatro repetições cada. As áreas experimentais para o terraceamento mecanizado nas propriedades $\mathrm{P} 1$, P2 e P3, apresentavam declividade entre 13 e $16 \%$ e estavam sendo utilizadas predominantemente como pastagem. Os terraços foram definidos e padronizados de base estreita, onde foram construídos com oito passadas de arado de discos, demarcadas previamente com aparelho de nível ótico, mira métrica e estacas.

Os arados de discos e tratores utilizados para o terraceamento em P1, P2 e P3 foram os disponíveis em cada uma, sendo identificados e discriminados nas Tabelas 1 e 2, respectivamente.

O desempenho operacional dos conjuntos trator/arado foi determinado pelos cálculos de eficiência operacional (Equação 1) e capacidade de campo efetiva (Equação 2), sendo os dados 
obtidos por estudo de tempos e movimentos de cada um, conforme metodologia de cronometragem de tempo contínuo descrita por Mialhe (1974). A metodologia consiste em realizar as medições sem que o cronômetro seja parado, a leitura do cronômetro é efetuada a cada vez que ocorre um ponto de medição, anotando o horário e o nome da atividade parcial concluída. O tempo de cada atividade parcial foi calculado através da subtração entre horário em que a atividade terminou e o horário que a mesma se iniciou durante a construção de quatro terraços consecutivos.

Tabela 1 Arados utilizados, suas características e valor de aquisição

\begin{tabular}{ccccccc}
\hline \multicolumn{7}{c}{ Arados de discos } \\
\hline Propriedade & Marca/Modelo $^{1}$ & $\begin{array}{c}\mathrm{N}^{\circ} \text { de } \\
\text { discos }\end{array}$ & $\begin{array}{c}\text { Diâmetro dos } \\
\operatorname{discos}^{2}(\mathrm{Pol})\end{array}$ & $\begin{array}{c}\text { Espaçamento x } \\
\text { Largura (mm) }\end{array}$ & $\begin{array}{c}\text { Peso } \\
(\mathrm{kg})\end{array}$ & $\begin{array}{c}\text { Valor de }_{\text {aquisição }}^{3} \\
(\mathrm{R} \$)\end{array}$ \\
\hline P1 & Baldan/Fixo & 2 & 28 & $570 \times 600$ & 314 & $3.000,00$ \\
P2 & Lavrale/Reversível & 2 & 28 & $570 \times 600$ & 261 & $3.900,00$ \\
P3 & Tatu/Fixo & 3 & 28 & $570 \times 900$ & 492 & $5.700,00$ \\
P3 & Tatu/Reversível & 3 & 28 & $550 \times 900$ & 506 & $8.790,00$ \\
\hline
\end{tabular}

${ }^{1}$ Modelos de acoplamento montado. ${ }^{2}$ Discos recortados. ${ }^{3}$ Valor estimado de mercado no estado de São Paulo no ano 2017.

Tabela 2 Tratores de pneus utilizados, suas características e valor de aquisição

\begin{tabular}{cccccc}
\hline \multicolumn{5}{c}{ Tratores de pneus } \\
\hline Propriedade & Marca/Modelo & Tração & Potência $(\mathrm{kW})$ & Ano de fabricação & Valor de aquisição $(\mathrm{R} \$)^{1}$ \\
\hline P1 & Agrale 5060 & $4 \times 2$ & 44,0 & 1997 & $24.000,00$ \\
P2 & Massey 255 & $4 \times 2$ & 36,7 & 1981 & $26.000,00$ \\
P3 & Massey 265 & 4 x 2 TDA & 63,3 & 2000 & $31.000,00$ \\
\hline
\end{tabular}

${ }^{1}$ Valor estimado de mercado no estado de São Paulo no ano de 2017.

$E \mathrm{f}=\frac{\mathrm{Tp}}{\mathrm{Tp}+\mathrm{Tm}} \times 100$

(Equação 1)

Em que: Ef é eficiência operacional dada em $\%$, Tp é o tempo produtivo do conjunto realizando a inversão de leiva e terraceamento propriamente dito, e Tm é o tempo de manobra, ambos dados em minutos.

Cce $=\frac{60 \mathrm{~min}}{\mathrm{Tp}+\mathrm{Tm}}$

(Equação 2)

Em que: Cce é a capacidade de campo efetiva dada em terraços produzidos por hora.

Cada terraço foi dimensionado com $100 \mathrm{~m}$ de comprimento espaçados em $30 \mathrm{~m}$, sendo considerado concluído ao final da oitava passada do conjunto trator/arado, sendo cada terraço uma repetição. As atividades consideradas para distinção dos tempos foram manobras de cabeceira, realizadas a aproximadamente $2 \mathrm{~km} \mathrm{~h}^{-}$ 1 e incluso o tempo de deslocamento entre terraços $(30 \mathrm{~m})$, e terraceamento propriamente dito (inversão de leiva e levantamento do terraço) realizado a $4 \mathrm{~km} \mathrm{~h}^{-1}$.
Os cálculos de custos mecanizados para produção dos terraços foram realizados como base na metodologia desenvolvida pela American Society of Agricultural Engineers (ASAE, 2002). Os custos foram compostos e organizados por custos fixos e variáveis, constituídos por custo fixo horário, custos com reparos e manutenção, depreciação, fator de alojamento seguros e taxas (FAST), mão-de-obra e combustível.

Para calcular o custo fixo horário do maquinário trator e arado, foram considerados os seus valores iniciais de aquisição com cotações realizadas na região de Avaré-SP. A vida útil e a quantidade de horas trabalhadas de cada equipamento foram obtidas segundo definições da ASAE (2002).

$\mathrm{O}$ valor da taxa de juros foi definido em 7,5\% ao ano, baseado no programa governamental Moderfrota de 2017, e o FAST em 0,5\% ao ano. O salário mensal do operador foi determinado em $\mathrm{R} \$ 1450,00$, com acréscimo de 96,27\% de encargos sociais $\left(13^{\circ}\right.$ salário, férias e INSS) e 176 horas mensais de trabalho. 
Para os cálculos de custos foram utilizadas as Equações 3 a 10:

$$
\mathrm{COT}=\frac{\mathrm{CH}}{\mathrm{Cce}}
$$

Em que: COT é o custo operacional por terraço dado em $\mathrm{R} \$$ terraço $^{-1}$, $\mathrm{CH}$ é custo horário com o maquinário dado em $\mathrm{R} \$ \mathrm{~h}^{-1}$ e Cce é capacidade de campo efetiva dada em terraços produzidos por hora $\left(\right.$ terraços $\left.h^{-1}\right)$.

$$
\begin{array}{ll}
\mathrm{CH}=\mathrm{CFH}+\mathrm{CC}+\mathrm{CRM}+\mathrm{CMO} & (\text { Equação 4) } \\
\mathrm{CFH}=\frac{\mathrm{CFA}}{\mathrm{NHTa}} & (\text { Equação 5) } \\
\mathrm{CFA}=\mathrm{Vi}[\mathrm{Dppa}+(\mathrm{Ja} \times \mathrm{i})+\mathrm{FAST}+\mathrm{Vi}] & (\text { Equação 6) } \\
\mathrm{NHTa}=\frac{\mathrm{Vuh}}{\mathrm{Vua}} & (\text { Equação 7) } \\
\mathrm{CC}=\mathrm{Ch} \times \mathrm{Pc} & (\text { Equação 8) } \\
\mathrm{CRM}=\mathrm{FRM} \times \frac{\mathrm{Vi}}{\mathrm{Vuh}} & (\text { Equação 9) } \\
\mathrm{CMO}=\frac{\mathrm{S}+\mathrm{E}}{\mathrm{NHTm}} & (\text { Equação 10) }
\end{array}
$$

Em que: CFH é o custo fixo horário em $\mathrm{R} \$ \mathrm{~h}^{-}$ ${ }^{1}, \mathrm{CC}$ é o custo com combustível em $\mathrm{R} \$ \mathrm{~h}^{-1}, \mathrm{CRM}$ é o custo com reparos e manutenção em $R \$ h^{-1} \mathrm{e}$ CMO é o custo da mão-de-obra do operador em $\mathrm{R} \$ \mathrm{~h}^{-1}$. CFA é o custo fixo anual em $\mathrm{R} \$ \mathrm{ano}^{-1}$, NHTa é o número de horas trabalhadas por ano, Vi é o valor inicial para aquisição em $\mathrm{R} \$$, Dpa é a depreciação anual em $\mathrm{R} \$$, Ja é a taxa de juro anual, i é a taxa de juros em \%, FAST é o fator de alojamentos, seguros e taxas em \%, Vuh é a vida útil em horas da máquina, Vua é a vida útil em anos da máquina, Ch é o consumo horário de combustível em $\mathrm{L} \mathrm{h}^{-1}$, Pc é o preço do combustível de $\mathrm{R} \$ 3,20 \mathrm{~L}^{-1}$, FRM é o fator percentual de reparos e manutenção de cada máquina e CMO é o custo da mão-de-obra do operador em $\mathrm{R} \$ \mathrm{~h}^{-1}$, S é o salário mensal em $\mathrm{R} \$$, E é o percentual de encargos trabalhistas mensais em \% e NHTm são as horas mensais de trabalho.

O consumo horário de combustível foi determinado por meio de um fluxômetro marca Flowmate oval, modelo Oval M-III, com precisão de 0,01 mililitros, instalado próximo ao filtro de combustível dos tratores. O equipamento registra uma unidade de pulso a cada mililitro de combustível que passa pelo mesmo e os valores de consumo horário de combustível são obtidos pelo produto da quantidade de pulsos registrados e o tempo gasto para percorrer o terraço e a manobra, separadamente, por meio da Equação 11.

$\mathrm{GH}=\frac{\Sigma \mathrm{p} \times 3,6}{\Delta \mathrm{t}}$

(Equação 11)

Em que: GH é o consumo horário de combustível em L h ${ }^{-1}, \Sigma$ p é o somatório de pulso, equivalente ao somatório de mililitros de combustível gasto para percorrer o percurso, $\Delta \mathrm{t}$ é o tempo gasto para percorrer o percurso em segundos e 3,6 é o fator de conversão.

Os resultados de desempenho operacional obtidos foram submetidos à análise de variância a $5 \%$ de significância e as médias foram comparadas pelo teste de Tukey a 5\% de probabilidade de erro. Os resultados referentes à receita e ao custo de produção foram analisados através de estatística descritiva.

\section{Resultados e Discussão}

Na Tabela 3 são apresentados os resultados de tempo demandados para produção de terraço com diferentes arados de discos, suas eficiências operacionais e capacidade de campo efetiva.

Entre os arados estudados não houve diferenças de tempo produtivo, ou seja, os minutos necessários para realizar a inversão de leiva e construção do terraço propriamente dito foi semelhante. Esse resultado foi ocasionado pela padronização do número de passadas e velocidade operacional dos conjuntos tratorarado, os quais apresentaram tempo produtivo médio em passadas de 9,15 min. Além disso a similaridade entre os terrenos operados proporcionaram aos diferentes conjuntos iguais dificuldades em inverter leiva e levantar terraços.

Diferentemente dos resultados de tempos produtivo os resultados de tempos de manobras foram diferenciados entre arados fixos (AF2D e AF3D) e reversíveis (AR2D e AR3D). Os arados fixos apresentaram maior tempo de manobras, em média 12,6 min, sendo aproximadamente 59,5\% maior que o tempo demandado com os reversíveis. Este resultado corrobora com Balastreire (2005), que descreve arados reversíveis sendo mais ágeis no momento das manobras, reduzindo o tempo demandado por estas e elevando a eficiência operacional. 
Desempenho operacional e econômico do terraceamento de pequenas propriedades com arados ...

Correia et al.

Tabela 3 Tempos demandados, eficiência operacional (Ef) e capacidade de campo efetiva (Cce) para construção de terraço com arados de discos

\begin{tabular}{lcccc}
\hline \multirow{2}{*}{ Arado } & \multicolumn{2}{c}{ Tempos demandados por terraço $(\mathrm{min})$} & \multirow{2}{*}{ Ef $(\%)$} & \multirow{2}{*}{ Cce $\left(\right.$ terraço $\left.\mathrm{h}^{-1}\right)$} \\
\cline { 2 - 3 } & Produtivo & Manobra & & \\
\hline AF3D & $9,0 \mathrm{a}$ & $12,0 \mathrm{a}$ & $44 \mathrm{~b}$ & $2,9 \mathrm{~b}$ \\
AF2D & $9,6 \mathrm{a}$ & $13,2 \mathrm{a}$ & $43 \mathrm{~b}$ & $2,8 \mathrm{~b}$ \\
AR3D & $8,4 \mathrm{a}$ & $5,4 \mathrm{a}$ & $59 \mathrm{a}$ & $4,4 \mathrm{~b}$ \\
AR2D & $9,6 \mathrm{a}$ & $4,8 \mathrm{~b}$ & $67 \mathrm{a}$ & $4,2 \mathrm{a}$ \\
\hline Média & 9,15 & 3,85 & 5,32 & 3,57 \\
CV $(\%)$ & 23,80 & 26,37 & 10,61 & 17,93 \\
DMS (5\%) & 4,8 & 4,8 & 12 & 1,35 \\
F tratamentos & $0,25^{\text {NS }}$ & $13,46^{* *}$ & $17,88^{*}$ & $6,62 * *$ \\
GL resíduos & 12 & 12 & 12 & 12 \\
\hline
\end{tabular}

AF3D: arado fixo de três discos, AF2D: arado fixo de dois discos, AR3D: arado reversível de três discos, AR2D: arado reversível de dois discos, CV (\%): coeficiente de variação, DMS (5\%): diferença mínima significativa, GL: grau de liberdade. Letras minúsculas iguais na coluna indica significância entre médias ao nível de $5 \%$ de probabilidade de erro pelo teste de Tukey.

AR2D e AR3D apresentaram maior eficiência operacional que AF2D e AF3D. Os modelos reversíveis apresentaram eficiência média de $63 \%$ e foram aproximadamente $20 \%$ mais eficientes que os fixos, resultado que pode ser compreendido devido o menor tempo demandado com manobras. Em comprovação a isso, os resultados de Cce foram maiores com os arados reversíveis AR2D e AR3D, produzindo em média 4,3 terraços $\mathrm{h}^{-1}$, produtividade $33,7 \%$ maior que a média obtida pelos fixos AF2D e AF3D.

Os resultados de consumo de combustível dos arados na operação de terraceamento são apresentados na Tabela 4.

Tabela 4 Consumo horário de combustível (Chc), consumo de combustível por terraço produzido (Cct), consumo de combustível por tempo produtivo (CcTp) e consumo de combustível por tempo de manobra (CcTm)

\begin{tabular}{lcccc}
\hline \multicolumn{1}{c}{ Arado } & Chc $\left(\mathrm{L} \mathrm{h}^{-1}\right)$ & $\mathrm{Cct}\left(\mathrm{L} \mathrm{terraç \textrm {O } ^ { - 1 }}\right)$ & $\mathrm{CcTp}\left(\mathrm{L} \mathrm{h}^{-1}\right)$ & $\mathrm{CcTm}\left(\mathrm{L} \mathrm{h}^{-1}\right)$ \\
\hline AF3D & $13,70 \mathrm{a}$ & $5,11 \mathrm{a}$ & $5,63 \mathrm{~b}$ & $7,62 \mathrm{a}$ \\
AF2D & $8,29 \mathrm{c}$ & $2,36 \mathrm{c}$ & $3,50 \mathrm{c}$ & $4,50 \mathrm{~b}$ \\
AR3D & $12,30 \mathrm{~b}$ & $3,70 \mathrm{~b}$ & $7,59 \mathrm{a}$ & $5,16 \mathrm{~b}$ \\
AR2D & $7,11 \mathrm{~d}$ & $1,69 \mathrm{c}$ & $4,69 \mathrm{~b}$ & $2,33 \mathrm{c}$ \\
\hline CV $(\%)$ & 5,01 & 11,88 & 11,00 & 15,10 \\
DMS (5\%) & 1,09 & 0,80 & 1,27 & 1,55 \\
F tratamentos & $147,38^{* *}$ & $63,01^{* *}$ & $32,29 * *$ & $34,66^{* *}$ \\
GL resíduos & 12 & 12 & 12 & 12 \\
\hline
\end{tabular}

AF3D: arado fixo de três discos, AF2D: arado fixo de dois discos, AR3D: arado reversível de três discos, AR2D: arado reversível de dois discos, CV (\%): coeficiente de variação, DMS (5\%): diferença mínima significativa, GL: grau de liberdade. Letras minúsculas iguais na coluna indica significância entre médias ao nível de $5 \%$ de probabilidade de erro pelo teste de Tukey.

$\mathrm{O}$ Che foi diferenciado entre os quatro arados estudados, sendo o AF3D o que apresentou maior Chc, 13,7 L h ${ }^{-1}$. Em relação ao AR3D, o Chc do AF3D foi 10,2\% maior. Comparando o Che desse arado com o menor obtido pelo AR2D, 7,11 L h' ${ }^{1}$, o consumo foi $48,1 \%$ maior.

Devido ao maior Che e a reduzida Cce o arado AF3D apresentou também maior Cct, 5,11 L terraço $^{-1}$ construído, consumo $66,9 \%$ maior que consumo de 1,69 $\mathrm{L}_{\text {terraço }}{ }^{-1}$ obtido pelo AR2D e 27,5\% maior que o Cct obtido pelo arado AR3D, também de três discos.

Com base nos resultados de consumo horário de combustível em tempos produtivo (CcTp), o arado AR3D apresentou maior consumo, 7,59 L $\mathrm{h}^{-1}$, significando que $61,7 \%$ do seu Che foi consumido em tempos produtivos de inversão de leiva e construção propriamente dita de terraços. 
O menor CcTP foi obtido pelo AF2D, 3,5 $\mathrm{L} \mathrm{h}^{-1}$, indicando que maior parte do seu Chc foi realizado nas manobras, o CcTm. AR2D e AF3D obtiveram CcTp semelhantes, 4,96 e 5,93 $\mathrm{L} \mathrm{h}^{-1}$ respectivamente.

Em relação ao consumo horário de combustível em tempos de manobra (CcTm), o arado AR2D apresentou menor consumo, 2,33 L $\mathrm{h}^{-1}$, significando que apenas $32,8 \%$ do seu Che foi consumido em manobras de cabeceira. $\mathrm{O}$ arado AR2D foi ágil suficiente para demandar reduzido tempo em manobras e elevada eficiência operacional, justificando o baixo CcTm. O maior CcTm foi obtido pelo AF3D, 7,62 $\mathrm{L} \mathrm{h}^{-1}$, indicando que $55,6 \%$ do seu Chc foi em manobras, comprovado pelo elevado tempo demandado para realização de manobras de cabeceira e ineficiência produtiva dos arados fixos.

Comparando os resultados de CcTm entre os arados de três discos o consumo foi $32,2 \%$ menor com o AR3D, devido a reversibilidade reduzir tempo de manobra e elevar a eficiência operacional. Comprando o CcTm entre os arados de dois discos a diferença também foi favorável ao modelo AR2D, cujo o consumo foi $48,2 \%$ menor que o AF2D.

Os resultados de custo horário $(\mathrm{CH})$, custo operacional por terraço (COT) e eficiência econômica (EFec) da construção de terraços com arados são apresentados na Figura 1.

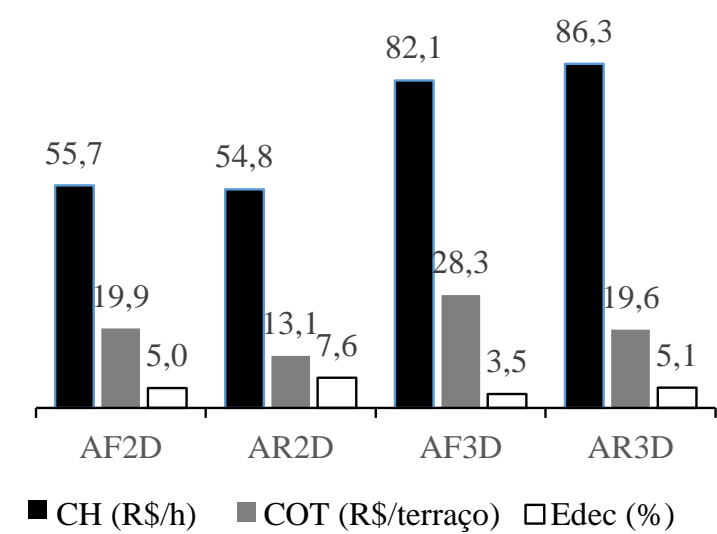

Figura 1 Custo horário, operacional e eficiência econômica da construção de terraços com arados.

$\mathrm{O}$ menor $\mathrm{CH}$ foi obtido com arado AR2D, $\mathrm{R} \$ 54,82 \mathrm{~h}^{-1}$, valor $1,6 \%$ menor que o outro arado de dois discos e $34,8 \%$ menor que o $\mathrm{CH}$ médio dos arados de três discos. Esse resultado pode ser compreendido devido ao menor Che verificado no AR2D.

Maior $\mathrm{CH}$ foi obtido com arado AR3D, $\mathrm{R} \$ 86,25 \mathrm{~h}^{-1}$, sendo $4,8 \%$ maior que AF3D e $35,9 \%$ maior que o $\mathrm{CH}$ média dos arados de dois discos. Devido os arados de três discos terem sido tracionados com o mesmo trator o maior $\mathrm{CH}$ do AR3D deve-se ao seu maior custo de aquisição, sendo aproximadamente $35 \%$ maior que o modelo fixo AF3D.

Em termos de COT o maior valor, $\mathrm{R} \$ 28,30$ terraço $^{-1}$, foi verificado com o AF3D. Esse custo foi respectivamente 30,$7 ; 29,6$ e $53,8 \%$ maior que o obtido com os arados AR3D, AF2D e AR2D. A obtenção de menor COT foi verificada com AR2D, devido ter apresentado menor $\mathrm{CH}$ e maior Cce.

Também em função de maior Cce e menor $\mathrm{CH}$ foi possível verificar maior EFec, de 7,6\%, ao arado AR2D. Esse resultado indica que para o sistema de terraceamento adotado o arado reversível de dois discos exigiu reduzido investimento para aquisição, baixo $\mathrm{CH}$ e elevada Cce, similar ao modelo reversível de três discos. A EFec obtida significa que para cada $R \$ 1,00$ investido na forma de $\mathrm{CH}$, produziu-se 7,6\% de terraços por hora com AR2D.

\section{Conclusão}

Pelos resultados obtidos e nas condições em que foi desenvolvido este trabalho, conclui-se que:

Independentemente do número de discos os arados reversíveis proporcionaram maior eficiência operacional e capacidade de campo efetiva.

Maior consumo horário e por terraço de combustível ocorreu com arado fixo de três discos.

O arado reversível de dois discos proporcionou menor custo horário, operacional e maior eficiência econômica, sendo este recomendado para pequenas propriedades rurais.

\section{Referências}

ASAE (AMERICAN SOCIETY OF AGRICULTURAL ENGINEERS). ASAE St28,3andards 2002: Estimating farm machinery costs. Iowa State University: standard A3-29, 2002. p. 1-9. 
Balastreire, L. A. Máquinas agrícolas. São Paulo: Manole, 2005. 310p.

CUNHA, F. F.; LEAL, A. J. F.; ROQUE, C. G. Planejamento de sistemas de terraceamento utilizando o software Terraço 3.0. Brazilian Geographical Journal: Geosciences and Humanities research medium, v.2, n.1, p.182196 , 2011. http://www.seer.ufu.br/index.php/braziliangeojournal/arti cle/view/11578

Dechen, S. C. F.; Telles, T. S.; Guimarães, M. F.; Maria, I. C. de. Perdas e custos associados à erosão hídrica em função de taxas de cobertura do solo. Bragantia, v.74, n.2, p.224-233, 2015. http://dx.doi.org/10.1590/1678-4499.0363

Ferreira, A. O.; Gonzatto, R.; Miola, A.; Eltz, F. L. F.; Amado, Telmo J. C. Influência da declividade e de níveis de cobertura de solo no processo de erosão com chuva simulada. Revista Verde de Agroecologia e Desenvolvimento Sustentável, v.5, n.5, p.182190 , 2010. http://www.gvaa.com.br/revista/index.php/RVADS/articl e/view/432

Magalhães, G. M. F. Análise da eficiência de terraços de retenção em sub-bacias hidrográficas do Rio São Francisco. Revista Brasileira de Engenharia Agrícola e Ambiental, v.17, n.10, p.1109-1115, 2013. http://dx.doi.org/10.1590/S1415-43662013001000013
Melo, R. P.; Albiero, D.; Praciano, A. C.; Cavalcante, E. S.; Fernandes, F. R. B. Análise do controle de qualidade de um terraceador trabalhando em um argissolo vermelho amarelo. Brazilian Journal of Biosystems Engineering, v.10, n.2, p.210-216, 2016. http://seer.tupa.unesp.br/index.php/BIOENG/article/view/ $\underline{393 / 283}$

Mialhe, L. G. Manual de mecanização agrícola. São Paulo: Ceres, 1974. 301p.

Panachuki, E.; Alves Sobrinho, T.; Vitorino, A. C. T.; Carvalho, D. F. D.; Urchei, M. A. Parâmetros físicos do solo e erosão hídrica sob chuva simulada, em área de integração agricultura-pecuária. Agriambi, v.10, n.2, p.261-268, 2006. http://dx.doi.org/10.1590/S1415$\underline{43662006000200003}$

Silva, R. P.; Voltarelli, M. A. Controle de qualidade em operações agrícolas. $1^{\mathrm{a}}$ ed. Jaboticabal: SBEA, 2015. 244p.

Vasconcelos, K. S. L.; Silva, T. J. J.; Melo, S. R. S. Mecanização da agricultura: demanda por tratores de rodas e máquinas agrícolas nos estados da região nordeste. Revista em Agronegócios e Meio Ambiente, v.6, n.2, p.207-222, 2013. http://periodicos.unicesumar.edu.br/index.php/rama/articl e/view/2371 